\title{
Spectrally Resolved Maker Fringes in High-Order Harmonic Generation
}

\author{
C. M. Heyl, ${ }^{1,2}$ J. Güdde, ${ }^{1}$ U. Höfer, ${ }^{1,3}$ and A. L'Huillier ${ }^{2}$ \\ ${ }^{1}$ Fachbereich Physik, Philipps-Universität Marburg, D-35032 Marburg, Germany \\ ${ }^{2}$ Department of Physics, Lund University, Post Office Box 118, SE-22100 Lund, Sweden \\ ${ }^{3}$ Donostia International Physics Centre (DIPC), Paseo de Manuel Lardizabal 4, 20018 San Sebastian, Spain
}

(Received 21 February 2011; published 12 July 2011)

\begin{abstract}
We investigate macroscopic interference effects in high-order harmonic generation using a Ti:sapphire laser operating at a $100 \mathrm{kHz}$ repetition rate. The structure and behavior of spectral and spatial interference fringes are explained and analytically described by transient phase matching of the long electron trajectory contribution. Time-frequency mapping due to the temporal chirp of the harmonic emission allows us to observe Maker fringes directly in the spectral domain.
\end{abstract}

DOI: 10.1103/PhysRevLett.107.033903

PACS numbers: 42.65.Ky, 42.65.Re

High-order harmonic generation (HHG) in gases is nowadays a well established method to generate coherent, extreme ultraviolet (XUV) radiation down to the attosecond time scale [1,2]. The rich physics behind HHG, which combines atoms in strong laser fields and nonlinear optics, has been progressively unraveled during the last 20 years. On the single-atom level, HHG is well described by a semiclassical three-step model: An electron wave packet leaves the atomic potential via tunneling, is accelerated by the external laser field, and recombines back to the ground state, thus emitting a burst of XUV radiation [3,4]. Two families of electron trajectories with different excursion times in the continuum contribute to the harmonic emission. These electron trajectories, or quantum paths, are called "short" and "long," reflecting the duration of the excursion in the continuum.

Interference phenomena are intimately connected to HHG, determining the harmonic emission both on the single-atom level as well as macroscopically. Interferences between consecutive attosecond bursts lead to harmonic frequency combs, while macroscopic interferences between the fields emitted at different positions in the medium determine the efficiency of the frequency conversion process. The latter effect is commonly used to select a dominant quantum path. When the contributions of short and long trajectories become comparable and overlap, quantum path interferences (QPIs) may occur [5-9]. Such interference effects provide a unique tool for probing the single-atom dipole and may allow direct access to atomic or molecular structures and dynamics.

This Letter reports on interference effects occurring in the emission of individual harmonics. A high repetition rate laser system allows us to measure complex interference phenomena with high signal/noise ratio. We observe

Published by the American Physical Society under the terms of the Creative Commons Attribution 3.0 License. Further distribution of this work must maintain attribution to the author(s) and the published article's title, journal citation, and DOI. interferences both in the harmonic spectra as a function of intensity and in the spatial far-field profiles. Similar effects have been previously observed and interpreted as QPIs $[5,7,10-18]$. Using an analytical model as well as numerical simulations, we show that these interferences occur due to the temporal variation of the phase matching conditions $[19,20]$ for only one (the long) quantum path. The frequency modulation (chirp) of the emitted radiation leads to a mapping between time and frequency $[5,21,22]$, which makes it possible to observe temporal Maker fringes directly in the spectral domain [23]. This new interpretation allows us to identify a common origin for several effects often observed in HHG such as spectral [5,7,10-16,25,26] and spatial [11,17] splitting, intensity modulation [7,13,18,27-29], and chirp dependence [5,30].

Generally, the yield $S_{q}$ of harmonic order $q$ can be expressed as

$$
S_{q} \propto\left|\int_{V} P_{q}(\mathbf{r}) \exp (i \Delta \mathbf{k} \cdot \mathbf{r}) d \mathbf{r}\right|^{2} .
$$

$P_{q}(\mathbf{r})$ denotes the laser-induced polarization in the medium at frequency $q \omega$, where $\omega$ is the laser frequency. $\Delta \mathbf{k}$ is the wave vector mismatch between the generated field and $P_{q}(\mathbf{r})$. In a one-dimensional case, assuming a constant wave vector mismatch and a constant nonlinear polarization strength within a short interaction volume of length $L$, the above equation reduces to

$$
S_{q} \propto L^{2} \operatorname{sinc}^{2}\left(\frac{\Delta k L}{2 \pi}\right) .
$$

$S_{q}$ oscillates as a function of $\Delta k$, and/or $L$, leading to an interference pattern known as Maker fringes [31].

For HHG in a nonguiding medium, $\Delta k$ is the sum of the wave vector mismatch due to the intensity-dependent single-atom dipole phase, $\Delta k_{q}$, the geometrical wave vector mismatch due to focusing, $\Delta k_{g}$, and the difference in wave vectors at frequencies $q \omega$ and $\omega$, due to dispersion in the neutral medium and due to free electrons, $\Delta k_{d}$. The dispersion terms are small in our experimental conditions and will be neglected in the following argumen- 
tation. The single-atom dipole phase $\varphi_{q}$ can be calculated by considering the classical action along a specific quantum path (i.e., short or long trajectory) for harmonic order $q$ and is well approximated by $\varphi_{q}=-\alpha_{q} I$, where $I$ is the fundamental intensity [32]. Consequently, $\Delta k_{q}=$ $-\alpha_{q} \partial I / \partial z$. During the laser pulse, $I$ and therefore $\Delta k_{q}$ and $\Delta k$ vary with time. This leads to oscillations of the harmonic yield [33], i.e., Maker fringes in the time domain [34], as illustrated in the simulation presented in Fig. 1(a).

The intensity variation of the dipole phase does not only affect phase matching but also the spectral properties of the radiation. The instantaneous harmonic frequency during the pulse is given by $[21,33]$

$$
\Omega_{q}(t)=q \omega+\alpha_{q} \frac{\partial I(t)}{\partial t} .
$$

The first term denotes the central harmonic frequency, while the second term describes the variation of the instantaneous frequency due to the single-atom response. The phase modulation process effectively maps time to frequency [Fig. 1(b)], leading to a spectrally broadened harmonic pulse with a blueshifted leading edge and a redshifted trailing edge. Since the harmonic emission is confined to the central part of the light pulse, the mapping process is almost linear. The temporal modulation of the harmonic yield due to Maker fringes appears therefore as a spectral modulation [Fig. 1(c)] [24]. Both processes, the phase matching-induced modulation, as well as the effective mapping into the frequency domain, require $\alpha_{q}$ to be sufficiently large. Since $\alpha_{q} \approx 1-5 \times 10^{-14} \mathrm{~cm}^{2} / \mathrm{W}$ for the short trajectory and $\alpha_{q} \approx 20-25 \times 10^{-14} \mathrm{~cm}^{2} / \mathrm{W}$ for the long trajectory [35], Maker fringes are more easily observed for the long trajectory. For sufficiently long pulses

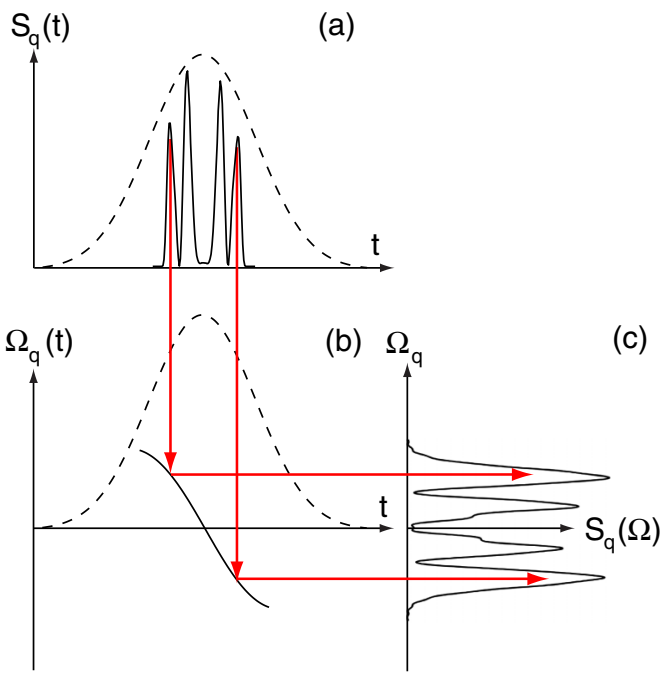

FIG. 1 (color online). Simulation of the mapping process for the 21st harmonic order: any temporal modulation of the harmonic signal $S_{q}(t)$ is mapped into the frequency domain due to a temporal modulation of the instantaneous harmonic frequency $\Omega_{q}(t)$. The dashed lines indicate the fundamental intensity profile. they do not depend on the laser carrier envelope phase (CEP) since both phase matching and time to frequency mapping are pure intensity effects. In conditions where consecutive harmonics overlap spectrally, an additional interference effect may appear which, however, washes out if the CEP is not stable [22,36].

In our experiments, we chose conditions where the long trajectory dominates the generation process. This implies a relatively tight focus geometry, as well as a positioning of the interaction cell close to the laser focus. We use a modified commercial Ti:sapphire laser system [37] delivering $45 \mathrm{fs}$ pulses with energies up to $7 \mu \mathrm{J}$ (without CEP stabilization). The laser beam ( $5 \mathrm{~mm}$ diameter) is focused by an achromatic lens $(f=60 \mathrm{~mm})$ into a continuous argon gas jet emitted from a nozzle with $100 \mu \mathrm{m}$ inner diameter. A concave XUV grating is used to spectrally separate the harmonic emission onto a microchannel plate assembly. Because of grazing incidence, the grating acts as a spatial window, selecting $\pm 11 \mathrm{mrad}$ in the horizontal direction (the total divergence is approximately $50 \mathrm{mrad}$ ). The microchannel plate records the spectrum in this direction and the central part ( $\pm 5 \mathrm{mrad}$ ) of the far-field profile in the vertical direction. The gas pressure inside the interaction cell is chosen to be relatively low (a few tens of mbar) to minimize dispersion and absorption effects. The pulse energy fluctuations of the fundamental laser field are less than $1 \%$ (rms deviation).

In Fig. 2(a) spatially integrated experimental spectra obtained with a gas jet at the focus (solid black line) and $\approx 0.2 \mathrm{~mm}$ behind the focus (dashed gray line) are shown. In the latter case, the harmonics appear intense and spectrally narrow, which is typical for the short trajectory emission. In contrast the harmonics generated at the focus are spectrally broad, as expected from the long trajectory, and exhibit interference structures. Figure 2(b) presents the corresponding spatially resolved spectrum, with clear splitting of the harmonic lines. The number of additional fringes on each side of the central frequencies $q \omega$ increases with intensity and decreasing harmonic order, as shown in Figs. 2(c) and 2(d). The fringes follow an almost symmetric, nested shape, similar to those observed in [7] under different experimental conditions.

We performed numerical simulations for a timedependent, three-dimensional harmonic source taking into account dispersion, absorption, and ionization effects. We used a simplified model for the single-atom response, deriving the phase of the dipole moment using classical trajectory calculations [3]. In accordance with previous experimental results, the amplitude of the dipole moment was assumed to scale as $I^{3 / 2}$ within the plateau [38]. The dependence of the dipole moment with harmonic order was adjusted to reproduce our experimental results. The numerical technique is a three-dimensional integration of Eq. (2) using Fourier-Hankel transform techniques. The integration in the far field is performed in accordance with the experimental geometry. Figure 2(d) presents the results 



FIG. 2 (color online). Experimental $(a-c)$ and simulated (d) harmonic spectra. (a) Spatially integrated signal measured when the gas jet was placed at the focus (black solid line) and $0.2 \mathrm{~mm}$ behind (gray dashed line; the signal strength was reduced by a factor of 0.05). (b) Far-field spatial profiles obtained at $5.2 \mu \mathrm{J}$ [white dashed line in (c)]. (c) and (d) show the experimental and simulated spatially integrated harmonic yield as a function of the driving pulse energy. The simulation only includes the long trajectory. (b-d) use the same normalized color scale as shown in (d).

of this simulation, considering only the contribution of the long trajectory. The experimentally observed interference patterns are well reproduced by the simulations.

The shape of the interference structures can be described analytically by examining the intensity dependence of the time-frequency mapping process. During the laser pulse phase matching is transiently optimized at certain intensities $I_{n}$, which are reached at given times $t_{n}$ depending on the peak intensity $I_{0}$. For a Gaussian intensity profile $I_{n}=I_{0} \exp \left(-t_{n}^{2} / \sigma^{2}\right)$ where $\sigma=\tau /(2 \sqrt{\ln 2})$ defines the temporal width with respect to the full width at half maximum $\tau$. Equation (3) can be evaluated for a certain $I_{n}$ leading to an equation for the instantaneous harmonic frequency $\Omega_{q}$ as a function of $I_{0}$ :

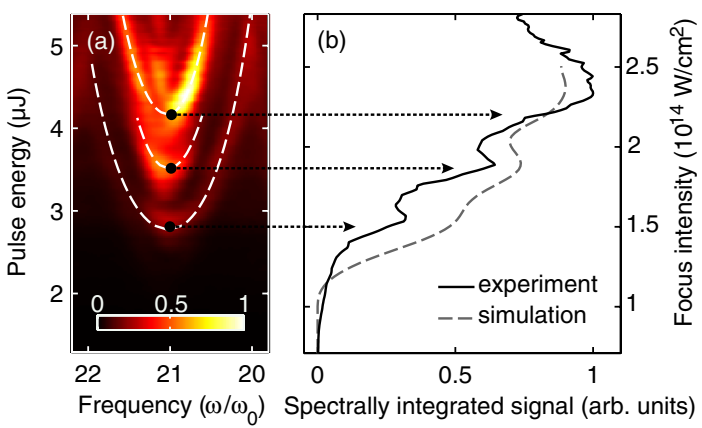

FIG. 3 (color online). Spectrally resolved (a) and integrated (b) harmonic signal as a function of the driving pulse energy for the 21st harmonic order. The white dashed lines are defined by Eq. (5), fitted to the experimental spectrum. Each line indicates a different transient phase matching intensity $I_{n}$.

$$
\Omega_{q}\left(I_{0}\right)=q \omega \pm \alpha_{q} \frac{I_{n}}{\sigma} 2 \sqrt{\ln \left(\frac{I_{0}}{I_{n}}\right)} .
$$

The equation can be inverted for direct comparison with the experimental spectrum as

$$
I_{0}\left(\Omega_{q}\right)=I_{n} \exp \left[\frac{\sigma^{2}\left(q \omega-\Omega_{q}\right)^{2}}{4 \alpha_{q}^{2} I_{n}^{2}}\right] .
$$

This equation describes the exponential shape of the maximum signal in the harmonic spectrum plotted as a function of the laser intensity ( $\propto$ pulse energy). It is indicated as dashed lines on the 21 st harmonic signal shown in Fig. 3(a), for different intensities $I_{n}$, adjusting $\alpha_{q}$ for best agreement with the experimental result. Calibrating the intensity reached in our experiment using the cutoff law which defines the maximum photon energy $E_{\max }=I_{p}+$ $3.17 U_{p}\left(I_{p}\right.$ and $U_{p}$ are the ionization potential and the ponderomotive energy, respectively), the energy axis can be converted approximately into an intensity axis. We found $\alpha_{17-25} \approx 40 \times 10^{-14} \mathrm{~cm}^{2} / \mathrm{W}$, which is consistent for the long trajectory, being slightly higher than the values reported in the literature [35].

A consequence of temporal Maker fringes is the modulation of the harmonic signal with intensity. Figure 3(b) shows the spectrally integrated signal as a function of the peak intensity. Both experimental and simulated signals exhibit modulations, due to interference maxima [Fig. 3(a)]. The modulation contrast is limited by spectral and spatial integration. Similar effects have been observed in the past [18,27-29] with various interpretations involving resonances and QPIs. In the case examined in the present work, their origin can be clearly assigned to temporal Maker fringes.

Finally, we calculate in Fig. 4 the spatial properties 4(a)-4(c) and the spectrum as a function of the laser pulse energy 4(d)-4(f) for the 21st harmonic in three different cases. Figures 4(a) and 4(d) refer to the case examined previously, i.e., taking into account only the long trajectory and a nonlinear medium with low density. The intensity dependence of phase matching leads to a similar effect in the spatial domain as in the temporal domain. A mapping 


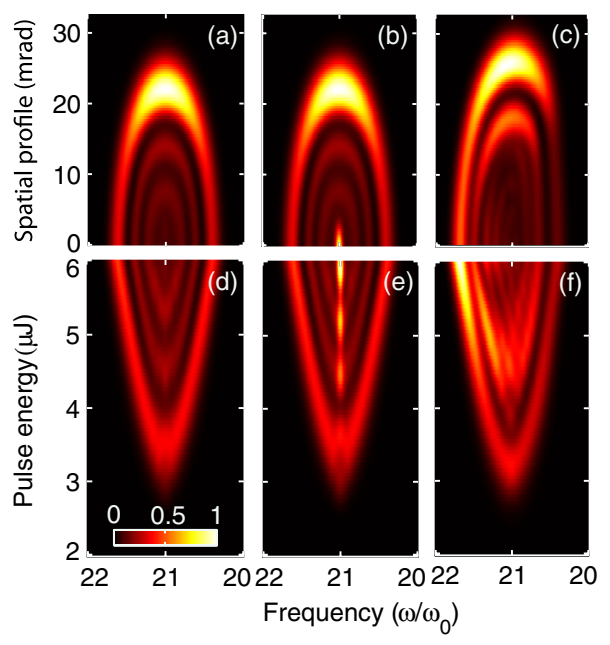

FIG. 4 (color online). (a-c) Spatially and spectrally resolved profiles for the 21 st harmonic order calculated for a pulse energy of $6 \mu \mathrm{J}$. (d-f) Spatially integrated (selecting $\pm 5 \mathrm{mrad}$ of the central part) signal as a function of the driving pulse energy. In ( $a, c)$ and (d, f) only the long trajectory is included; in (b) and (e) a contribution from the short trajectory is added. In (c) and (f) the pressure was changed from $50 \mathrm{mbar}$ (other figures) to 500 mbar and the intensity was slightly increased by setting the interaction medium closer to the focus.

between near-field and far-field profiles gives rise to phase matching rings in the far field. Figures 4(b) and 4(e) are calculated using the same conditions as for $4(\mathrm{a})$ and $4(\mathrm{~d})$, with the addition of the short trajectory. This leads to an increased yield and interferences between both trajectories at the center of the spectral and spatial profiles. A similar feature can be observed in our experimental data (Fig. 2) for the 15th and 17th harmonic. Since the short trajectory contribution leads to well collimated and spectrally narrow harmonics [39], interference effects observed outside these regions involve the long trajectory only.

In Figs. 4(c) and 4(f), we examine the influence of ionization in relation to a higher gas density. Equation (3) can be generalized to include the ionization-induced chirp of the fundamental field by adding on the right $-q \partial \varphi_{f} / \partial t$, where $\varphi_{f}$ denotes the phase of the fundamental pulse envelope, thus modifying the time to frequency mapping. Ionization also changes the phase matching conditions and reduces the number of contributing atoms on the trailing edge of the pulse [20]. Both effects lead to asymmetric temporal and spectral profiles. In general, the timefrequency mapping is modified when the fundamental field is chirped. A positive (negative) chirp decreases (increases) the spectral width of the individual harmonics.

In conclusion, we have identified spectral Maker fringes in HHG which originate from transient phase matching of the long trajectory contribution. This interference effect allows us to analyze and control the phase matching process as well as to spectrally and spatially tune the harmonic emission by changing the intensity and/or chirp of the fundamental pulse. The similarities between our results and previous experiments, where interference effects were interpreted in terms of QPIs, suggest that macroscopic effects have to be considered. In general, our results beautifully illustrate the fascinating interplay between macroscopic and microscopic effects in HHG.

We thank Pascal Salières for valuable discussions. This research was supported by the Deutsche Forschungsgemeinschaft through HO 2295/1-2 and GRK 790, the European Research Council (ALMA), the Marie Curie Training Network ATTOFEL, the Knut and Alice Wallenberg foundation, and the Swedish Research Council.

[1] F. Krausz and M. Ivanov, Rev. Mod. Phys. 81, 163 (2009).

[2] T. Popmintchev et al., Nat. Photon. 4, 822 (2010).

[3] P. B. Corkum, Phys. Rev. Lett. 71, 1994 (1993).

[4] K. C. Kulander, K. J. Schafer, and J. L. Krause, in SuperIntense Laser-Atom Physics (Plenum Press, New York, 1993).

[5] Z. Chang et al., Phys. Rev. A 58, R30 (1998).

[6] K. Schiessl et al., Phys. Rev. Lett. 99, 253903 (2007).

[7] A. Zaïr et al., Phys. Rev. Lett. 100, 143902 (2008).

[8] J. Tate et al., Phys. Rev. Lett. 98, 013901 (2007).

[9] M. V. Frolov, N. L. Manakov, and A. F. Starace, Phys. Rev. Lett. 100, 173001 (2008).

[10] H. Xu et al., Phys. Rev. A 78, 033841 (2008).

[11] E. Brunetti, R. Issac, and D. A. Jaroszynski, Phys. Rev. A 77, 023422 (2008).

[12] M. Holler et al., Opt. Express 17, 5716 (2009).

[13] E. Cormier et al., Eur. Phys. J. Special Topics 175, 191 (2009).

[14] T. Auguste et al., Phys. Rev. A 80, 033817 (2009).

[15] P. Liu et al., Phys. Rev. A 79, 063823 (2009).

[16] X. He et al., Phys. Rev. A 79, 063829 (2009).

[17] F. Schapper et al., Opt. Express 18, 2987 (2010).

[18] J. E. Kruse et al., Phys. Rev. A 82, 033438 (2010).

[19] S. Kazamias et al., Phys. Rev. Lett. 90, 193901 (2003).

[20] T. Pfeifer et al., Opt. Express 15, 17120 (2007).

[21] M. Gaarde et al., Phys. Rev. A 59, 1367 (1999).

[22] G. Sansone et al., Phys. Rev. Lett. 94, 193903 (2005).

[23] A similar effect has been observed in the third harmonic generation [24].

[24] I. A. Heisler et al., Appl. Phys. Lett. 92, 091109 (2008).

[25] Y.S. Wang et al., Phys. Rev. A 62, 063806 (2000).

[26] Z. Fangchuan et al., Phys. Rev. A 65, 033808 (2002).

[27] A. L'Huillier, P. Balcou, and L. A. Lompre, Phys. Rev. Lett. 68, 166 (1992).

[28] D. C. Yost et al., Nature Phys. 5, 815 (2009).

[29] J. Seres et al., Nature Phys. 6, 455 (2010).

[30] D. G. Lee et al., Phys. Rev. Lett. 87, 243902 (2001).

[31] P. D. Maker et al., Phys. Rev. Lett. 8, 21 (1962).

[32] M. Lewenstein, P. Salières, and A. L'Huillier, Phys. Rev. A 52, 4747 (1995).

[33] P. Salières et al., Adv. At. Mol. Opt. Phys. 41, 83 (1999).

[34] S. Kazamias et al., Phys. Rev. A 68, 033819 (2003).

[35] K. Varjú et al., J. Mod. Opt. 52, 379 (2005).

[36] E. Mansten et al., Phys. Rev. Lett. 102, 083002 (2009).

[37] C. M. Heyl et al. (unpublished).

[38] C. G. Wahlström et al., Phys. Rev. A 48, 4709 (1993).

[39] C. Lyngå et al., Phys. Rev. A 60, 4823 (1999). 